\title{
Research Article \\ Exact Solutions for a Third-Order KdV Equation with Variable Coefficients and Forcing Term
}

\author{
Alvaro H. Salas $\mathbf{S}^{1,2}$ and Cesar A. Gómez $\mathbf{S}^{3}$ \\ ${ }^{1}$ Department of Mathematics, Universidad de Caldas, Campus La Nubia, Manizales, \\ Caldas Cll 65 \# 26-10, A. A. 275, Colombia \\ ${ }^{2}$ Department of Mathematics and Statistics, National University of Colombia, \\ Manizales Cll 65, Palogrande Stadium, Colombia \\ ${ }^{3}$ Department of Mathematics, National University of Colombia, Bogotá, Colombia
}

Correspondence should be addressed to Alvaro H. Salas S, asalash2002@yahoo.com

Received 20 April 2009; Revised 14 August 2009; Accepted 31 August 2009

Recommended by Ji Huan He

The general projective Riccati equation method and the Exp-function method are used to construct generalized soliton solutions and periodic solutions to special $\mathrm{KdV}$ equation with variable coefficients and forcing term.

Copyright (C) 2009 A. H. Salas S and C. A. Gómez S. This is an open access article distributed under the Creative Commons Attribution License, which permits unrestricted use, distribution, and reproduction in any medium, provided the original work is properly cited.

\section{Introduction}

It is well known that in an early phase of the development of the solitons theory, there were already many applications in physics and engineering. In particular, traveling waves as solutions of the KdV equation

$$
u_{t}+6 u u_{x}+u_{x x x}=0
$$

have been of some interest since 150 years. Some generalizations of this last equation have been studied recently. For instance, the equation

$$
u_{t}+k_{1} t^{n} u u_{x}+k_{2} t^{m} u_{x x x}=0
$$

where $k_{1}, k_{2}$ are arbitrary constants, which have applications in physics, has been analyzed in [1] from the point of view of its exact solutions. The search of explicit solutions to 
nonlinear partial differential equations (NLPDEs) using analytic methods is not an easy task. However, the use of computational methods facilitates this work. Some powerful computational methods such as the tanh method [2], the generalized tanh method [3, 4], the extended tanh method [5-10], the improved tanh-coth method [11-13], the Exp-function method [14-18], the modified Exp-function method [19], the Cole-Hopf transformation [20], the projective Riccati equation method (PREM) [21, 22], the generalized projective Riccati equations method [23-25], the extended hyperbolic function method [26], and many other methods have been developed in this direction. The PREM and the Exp-function method have been used in a satisfactory form to solve some NLPDEs [15-17, 24, 25, 27-30]. In this paper, we use this last two methods to obtain soliton and periodic solutions to the following special $\mathrm{KdV}$ equation with variable coefficients and forcing term:

$$
u_{t}+\alpha(t) u u_{x}+k \alpha(t) u_{x x x}=F(t)
$$

where $F(t)$ is an external forcing function varying with time $t, k$ is a constant, and $\alpha=\alpha(t)$ is a function of $t, \alpha(t) \neq 0$. Equation (1.3) is a generalization of the following equation $[15,17,31]$ :

$$
u_{t}+\alpha u u_{x}+\beta u_{x x x}=F(t) \quad(\alpha, \beta=\text { const })
$$

which results from (1.3) by taking $\alpha=$ const and $k=\beta / \alpha$.

We suppose that the solution to (1.3) has the form

$$
u(x, t)=\int F(t) d t+v(x, t)
$$

Therefore, (1.3) reduces to

$$
v_{t}+\alpha(t) v v_{x}+\alpha(t) \int F(t) d t v_{x}+k \alpha(t) v_{x x x}=0
$$

Now we consider the transformation

$$
v=V(\xi), \quad \xi=\lambda x+\int h(t) d t
$$

where $\lambda$ is a constant and $h(t)$ is an unknown function of $t$ to be determined later. Substituting (1.7) into (1.6), we obtain

$$
h(t) V^{\prime}(\xi)+\alpha(t) \lambda(V(\xi)+f(t)) V^{\prime}(\xi)+k \lambda^{3} \alpha(t) V^{\prime \prime \prime}(\xi)=0,
$$

where

$$
f=f(t)=\int F(t) d t
$$




\section{The Exp-Function Method}

Recently $\mathrm{He}$ and $\mathrm{Wu}$ [14] have introduced the Exp-function method to solve nonlinear equations. In particular, the Exp-function method is a useful tool for solving nonlinear equations with high nonlinearity. The method has been used in a satisfactory way by other authors to solve a great variety of nonlinear wave equations [14-19]. The Exp-function method is very simple and straightforward and is based on a priori assumption that traveling wave solutions to a nonlinear partial differential equation in the form

$$
F\left(u, u_{x}, u_{t}, u_{x x}, u_{x t}, u_{t t}, \ldots\right)=0
$$

can be found using the expression

$$
u(\xi)=\frac{\sum_{n=-c}^{d} a_{n} \exp (n \xi)}{\sum_{n=-p}^{q} a_{m} \exp (m \xi)}
$$

where $c, d, p$, and $q$ are positive integers which could be freely chosen; $a_{n}$ and $b_{n}$ are unknown constants to be determined. According to this, we suppose that solutions to (1.8) can be expressed in the form

$$
v(\xi)=\frac{\sum_{n=-c}^{d} a_{n} \exp (n \xi)}{\sum_{m=-p}^{q} b_{m} \exp (m \xi)}=\frac{a_{-c} \exp (-c \xi)+\cdots+a_{d} \exp (d \xi)}{b_{-p} \exp (-p \xi)+\cdots+b_{q} \exp (q \xi)}
$$

where $c, d, p$, and $q$ are positive integers which are unknown to be determined later; $a_{n}$ and $b_{m}$ are unknown constants. We have the following two cases.

2.1. Case 1: $p=c=1$ and $d=q=1$

In this case, the trial solution to (1.8) becomes

$$
V(\xi)=\frac{a_{1} \exp (\xi)+a_{0}+a_{-1} \exp (-\xi)}{\exp (\xi)+b_{0}+b_{-1} \exp (-\xi)} .
$$

Substituting (2.4) into (1.8) we obtain a polynomial equation in the variable $\eta=\exp (\xi)$. Equating to zero the coefficients of all powers of $\eta$ yields a set of algebraic equations. Solving it with the aid of a computer, we get $a_{0}=b_{0}\left(a_{1}+6 k \lambda^{2}\right), a_{-1}=(1 / 4) a_{1} b_{0}^{2}, b_{-1}=b_{0}^{2} / 4$, and $h(t)=-\lambda \alpha(t)\left(a_{1}+f(t)+k \lambda^{2}\right)$, and, from (1.5), (1.7), and (2.3), one solution to (1.3) is given by

$$
u(x, t)=\frac{a_{1}\left(b_{0}+2 e^{\xi}\right)^{2}+24 b_{0} k \lambda^{2} e^{\xi}}{\left(b_{0}+2 e^{\xi}\right)^{2}}+\int F(t) d t
$$


where

$$
\xi=\xi(x, t)=\lambda x+\int h(t) d t=\lambda x-\int \lambda \alpha(t)\left(a_{1}+k \lambda^{2}+\int F(t) d t\right) d t
$$

and $a_{1}, b_{0}$, and $\lambda$ are arbitrary real or complex numbers.

2.2. Case 2: $p=c=2$ and $d=q=2$

In this case, the trial solution to (1.8) becomes

$$
V(\xi)=\frac{a_{2} \exp (2 \xi)+a_{1} \exp (\xi)+a_{0}+a_{-1} \exp (-\xi)+a_{-2} \exp (-2 \xi)}{\exp (2 \xi)+b_{1} \exp (\xi)+b_{0}+b_{-1} \exp (-\xi)+b_{-2} \exp (-2 \xi)}
$$

As in the first case, we obtain an algebraic system. Solving it gives

$$
\begin{aligned}
a_{0}= & -\frac{-2 a_{1} b_{1}\left(a_{2}+3 k \lambda^{2}\right)+a_{2}\left(a_{2} b_{1}^{2}+6\left(b_{1}^{2}-b_{0}\right) k \lambda^{2}\right)+a_{1}^{2}}{6 k \lambda^{2}}, \\
a_{-1}= & \frac{a_{1}-a_{2} b_{1}}{432 k^{3} \lambda^{6}}\left(-a_{1} b_{1}\left(27 a_{2} k \lambda^{2}+2 a_{2}^{2}+72 k^{2} \lambda^{4}\right)\right. \\
& \left.+\left(a_{2}+6 k \lambda^{2}\right)\left(12 a_{2} b_{1}^{2} k \lambda^{2}+a_{2}^{2} b_{1}^{2}+72 b_{0} k^{2} \lambda^{4}\right)+a_{1}^{2}\left(a_{2}+9 k \lambda^{2}\right)\right), \\
a_{-2}= & \frac{1}{6912 k^{4} \lambda^{8}} a_{2}\left(a_{1}-a_{2} b_{1}\right)^{2}\left(8 a_{2} b_{1}^{2} k \lambda^{2}-2 a_{1} b_{1}\left(a_{2}+4 k \lambda^{2}\right)+a_{2}^{2} b_{1}^{2}+a_{1}^{2}+48 b_{0} k^{2} \lambda^{4}\right), \\
b_{-1}= & \frac{1}{432 k^{3} \lambda^{6}}\left(a_{1}-a_{2} b_{1}\right)\left(9 a_{2} b_{1}^{2} k \lambda^{2}-a_{1} b_{1}\left(2 a_{2}+9 k \lambda^{2}\right)+a_{2}^{2} b_{1}^{2}+a_{1}^{2}+72 b_{0} k^{2} \lambda^{4}\right), \\
b_{-2}= & \frac{1}{6912 k^{4} \lambda^{8}}\left(a_{1}-a_{2} b_{1}\right)^{2}\left(8 a_{2} b_{1}^{2} k \lambda^{2}-2 a_{1} b_{1}\left(a_{2}+4 k \lambda^{2}\right)+a_{2}^{2} b_{1}^{2}+a_{1}^{2}+48 b_{0} k^{2} \lambda^{4}\right), \\
h(t)= & -\lambda \alpha(t)\left(a_{2}+f(t)+k \lambda^{2}\right) .
\end{aligned}
$$

From (1.5), (1.7), and (2.7) we may verify that to this set of values corresponds the solution

$$
\begin{aligned}
u(x, t)= & \frac{1}{\left(a_{1}-a_{2} b_{1}+12 k \lambda^{2} e^{\xi}\right)^{2}}\left(a_{1}^{2} a_{2}+a_{1}\left(-2 a_{2}^{2} b_{1}+24 a_{2} k \lambda^{2} e^{\xi}+144 k^{2} \lambda^{4} e^{\xi}\right)\right. \\
& \left.+a_{2}\left(a_{2}^{2} b_{1}^{2}-24 a_{2} b_{1} k \lambda^{2} e^{\xi}+144 k^{2} \lambda^{4} e^{\xi}\left(e^{\xi}-b_{1}\right)\right)\right) \\
& +\int F(t) d t,
\end{aligned}
$$


where

$$
\xi=\xi(x, t)=\lambda x+\int h(t) d t=\lambda x-\int \lambda \alpha(t)\left(a_{2}+k \lambda^{2}+\int F(t) d t\right) d t
$$

and $a_{1}, a_{2}, b_{1}$, and $\lambda$ are arbitrary real or complex numbers.

\section{General Projective Riccati Equation Method}

The projective Riccati equation method was introduced initially in [21] and generalizations of this method have been used in a satisfactory way by several authors to solve nonlinear partial equations [22-25]. Using this last method [24, 25, 27-30], we seek solutions to (1.8) in the form

$$
V(\xi)=a_{0}+\sum_{i=1}^{m} \sigma^{i-1}(\xi)\left(a_{i} \sigma(\xi)+b_{i} \tau(\xi)\right)
$$

where $a_{0}, a_{1}, b_{1}, \ldots$ are constants and $\sigma(\xi)$ and $\tau(\xi)$ satisfy the system

$$
\sigma^{\prime}(\xi)=\epsilon \sigma(\xi) \tau(\xi), \quad \tau^{\prime}(\xi)=R+\epsilon \tau^{2}(\xi)-\mu \sigma(\xi)
$$

In (3.2), $\epsilon= \pm 1$ and $R$ and $\mu$ are certain constants. These equations have following solutions.

Case 1. When $\epsilon=-1$ and $R \neq 0$,

$$
\begin{gathered}
\sigma_{1}(\xi)=\frac{R \operatorname{sech}(\sqrt{R} \xi)}{\mu \operatorname{sech}(\sqrt{R} \xi)+1}, \quad \tau_{1}(\xi)=\frac{\sqrt{R} \tanh (\sqrt{R} \xi)}{\mu \operatorname{sech}(\sqrt{R} \xi)+1} \\
\sigma_{2}(\xi)=\frac{R \operatorname{csch}(\sqrt{R} \xi)}{\mu \operatorname{csch}(\sqrt{R} \xi)+1}, \quad \tau_{2}(\xi)=\frac{\sqrt{R} \operatorname{coth}(\sqrt{R} \xi)}{\mu \operatorname{csch}(\sqrt{R} \xi)+1}
\end{gathered}
$$

Case 2. When $\epsilon=1$ and $R \neq 0$,

$$
\begin{array}{ll}
\sigma_{3}(\xi)=\frac{R \sec (\sqrt{R} \xi)}{\mu \sec (\sqrt{R} \xi)+1}, & \tau_{3}(\xi)=\frac{\sqrt{R} \tan (\sqrt{R} \xi)}{\mu \sec (\sqrt{R} \xi)+1}, \\
\sigma_{4}(\xi)=\frac{R \csc (\sqrt{R} \xi)}{\mu \csc (\sqrt{R} \xi)+1}, & \tau_{4}(\xi)=\frac{\sqrt{R} \cot (\sqrt{R} \xi)}{\mu \csc (\sqrt{R} \xi)+1} .
\end{array}
$$


Case 3. When $R=\mu=0$,

$$
\sigma_{5}(\xi)=\frac{C}{\xi}, \quad \tau_{5}(\xi)=\frac{1}{\epsilon \xi} .
$$

In this last case, we seek solutions to (1.8) in the form

$$
V(\xi)=\sum_{i=0}^{m} a_{i} \tau^{i}(\xi)
$$

where $\tau^{\prime}(\xi)=\tau^{2}(\xi)$. holds:

For any pair $(\sigma(\xi), \tau(\xi))$ of functions given by (3.3) or (3.4) the following equation

$$
\tau^{2}(\xi)=-\epsilon\left[R-2 \mu \sigma(\xi)+\frac{\mu^{2}-1}{R} \sigma^{2}(\xi)\right] .
$$

\subsection{Periodic and Soliton Solutions}

Periodic and soliton solutions are obtained when $R \neq 0$ and $\epsilon= \pm 1$ and it corresponds to the first two cases. Substituting (3.1), along with (3.2) and (3.7) into the left hand of (1.8) and collecting all terms with the same power in $\sigma^{i}(\xi) \tau^{j}(\xi)$, we get a polynomial in the variables $\sigma=\sigma(\xi)$ and $\tau=\tau(\xi)$. We may choose $m=2$. Thus, we seek solutions to (1.8) in the form

$$
V(\xi)=a_{0}+a_{1} \sigma(\xi)+b_{1} \tau(\xi)+\sigma(\xi)\left(a_{2} \sigma(\xi)+b_{2} \tau(\xi)\right)
$$

We equate each coefficient of the polynomial to zero. This will give an overdetermined system of algebraic equations involving the parameters $a_{i}, b_{i}(i=0, \ldots, m), \lambda$ and $\mu, R$, and the unknown function $h(t)$. Having determined these parameters, we may determine $V(\xi)$, and using (1.5) we obtain an exact solution $u(x, t)$ in a closed form. The corresponding system reads.

(i) $b_{1}^{2} R^{3} \alpha \lambda-b_{1}^{2} R^{3} \alpha \epsilon^{2} \lambda=0$.

(ii) $6 a_{1} k R \alpha \lambda^{3} \epsilon^{4}-6 a_{1} k R \alpha \lambda^{3} \mu^{2} \epsilon^{4}+48 a_{2} k R^{2} \alpha \lambda^{3} \mu \epsilon^{4}-3 b_{1} b_{2} R \alpha \lambda \mu^{2} \epsilon^{2}+3 b_{1} b_{2} R \alpha \lambda \epsilon^{2}-$ $18 a_{2} k R^{2} \alpha \lambda^{3} \mu \epsilon^{2}+4 b_{2}^{2} R^{2} \alpha \lambda \mu \epsilon^{2}+3 a_{1} a_{2} R^{2} \alpha \lambda \epsilon-b_{2}^{2} R^{2} \alpha \lambda \mu=0$.

(iii) $24 a_{2} k R \alpha \lambda^{3} \epsilon^{4}-24 a_{2} k R \alpha \lambda^{3} \mu^{2} \epsilon^{4}-2 b_{2}^{2} R \alpha \lambda \mu^{2} \epsilon^{2}+2 b_{2}^{2} R \alpha \lambda \epsilon^{2}+2 a_{2}^{2} R^{2} \alpha \lambda \epsilon=0$.

(iv) $6 b_{1} k \alpha \lambda^{3} \mu^{4} \epsilon^{5}+6 b_{1} k \alpha \lambda^{3} \epsilon^{5}-96 b_{2} k R \alpha \lambda^{3} \mu^{3} \epsilon^{5}-12 b_{1} k \alpha \lambda^{3} \mu^{2} \epsilon^{5}+96 b_{2} k R \alpha \lambda^{3} \mu \epsilon^{5}+$ $36 b_{2} k R \alpha \lambda^{3} \mu^{3} \epsilon^{3}-36 b_{2} k R \alpha \lambda^{3} \mu \epsilon^{3}-3 a_{2} b_{1} R \alpha \lambda \mu^{2} \epsilon^{2}-3 a_{1} b_{2} R \alpha \lambda \mu^{2} \epsilon^{2}+3 a_{2} b_{1} R \alpha \lambda \epsilon^{2}+$ $3 a_{1} b_{2} R \alpha \lambda \epsilon^{2}+8 a_{2} b_{2} R^{2} \alpha \lambda \mu \epsilon^{2}-a_{2} b_{2} R^{2} \alpha \lambda \mu=0$.

(v) $24 b_{2} k \alpha \lambda^{3} \mu^{4} \epsilon^{5}+24 b_{2} k \alpha \lambda^{3} \epsilon^{5}-48 b_{2} k \alpha \lambda^{3} \mu^{2} \epsilon^{5}-4 a_{2} b_{2} R \alpha \lambda \mu^{2} \epsilon^{2}+4 a_{2} b_{2} R \alpha \lambda \epsilon^{2}=0$, $-6 a_{1} k R^{3} \alpha \lambda^{3} \epsilon^{4}+5 a_{1} k R^{3} \alpha \lambda^{3} \epsilon^{2}-3 b_{1} b_{2} R^{3} \alpha \lambda \epsilon^{2}+2 b_{1}^{2} R^{2} \alpha \lambda \mu \epsilon^{2}+a_{0} a_{1} R^{2} \alpha \lambda \epsilon+a_{1} f R^{2} \alpha \lambda \epsilon+$ $a_{1} R^{2} h \epsilon+2 b_{1} b_{2} R^{3} \alpha \lambda-b_{1}^{2} R^{2} \alpha \lambda \mu=0$.

(vi) $-24 a_{2} k R^{3} \alpha \lambda^{3} \epsilon^{4}+12 a_{1} k R^{2} \alpha \lambda^{3} \mu \epsilon^{4}+16 a_{2} k R^{3} \alpha \lambda^{3} \epsilon^{2}-b_{1}^{2} R \alpha \lambda \mu^{2} \epsilon^{2}-2 b_{2}^{2} R^{3} \alpha \lambda \epsilon^{2}+b_{1}^{2} R \alpha \lambda \epsilon^{2}-$ $6 a_{1} k R^{2} \alpha \lambda^{3} \mu \epsilon^{2}+6 b_{1} b_{2} R^{2} \alpha \lambda \mu \epsilon^{2}+a_{1}^{2} R^{2} \alpha \lambda \epsilon+2 a_{0} a_{2} R^{2} \alpha \lambda \epsilon+2 a_{2} f R^{2} \alpha \lambda \epsilon+2 a_{2} R^{2} h \epsilon+$ $b_{2}^{2} R^{3} \alpha \lambda-2 b_{1} b_{2} R^{2} \alpha \lambda \mu=0$. 
(vii) $6 b_{1} k R^{4} \alpha \lambda^{3} \epsilon^{5}-8 b_{1} k R^{4} \alpha \lambda^{3} \epsilon^{3}-a_{0} b_{1} R^{3} \alpha \lambda \epsilon^{2}-b_{1} f R^{3} \alpha \lambda \epsilon^{2}-b_{1} R^{3} h \epsilon^{2}+2 b_{1} k R^{4} \alpha \lambda^{3} \epsilon+$ $a_{0} b_{1} R^{3} \alpha \lambda+b_{1} f R^{3} \alpha \lambda+b_{1} R^{3} h=0$.

(viii) $24 b_{2} k R^{4} \alpha \lambda^{3} \epsilon^{5}-24 b_{1} k R^{3} \alpha \lambda^{3} \mu \epsilon^{5}-28 b_{2} k R^{4} \alpha \lambda^{3} \epsilon^{3}+28 b_{1} k R^{3} \alpha \lambda^{3} \mu \epsilon^{3}-2 a_{1} b_{1} R^{3} \alpha \lambda \epsilon^{2}-$ $2 a_{0} b_{2} R^{3} \alpha \lambda \epsilon^{2}-2 b_{2} f R^{3} \alpha \lambda \epsilon^{2}+2 a_{0} b_{1} R^{2} \alpha \lambda \mu \epsilon^{2}+2 b_{1} f R^{2} \alpha \lambda \mu \epsilon^{2}-2 b_{2} R^{3} h \epsilon^{2}+2 b_{1} R^{2} \mu h \epsilon^{2}+$ $5 b_{2} k R^{4} \alpha \lambda^{3} \epsilon-5 b_{1} k R^{3} \alpha \lambda^{3} \mu \epsilon+a_{1} b_{1} R^{3} \alpha \lambda+a_{0} b_{2} R^{3} \alpha \lambda+b_{2} f R^{3} \alpha \lambda-a_{0} b_{1} R^{2} \alpha \lambda \mu-b_{1} f R^{2} \alpha \lambda \mu+$ $b_{2} R^{3} h-b_{1} R^{2} \mu h=0$.

(ix) $-12 b_{1} k R^{2} \alpha \lambda^{3} \epsilon^{5}+36 b_{1} k R^{2} \alpha \lambda^{3} \mu^{2} \epsilon^{5}-96 b_{2} k R^{3} \alpha \lambda^{3} \mu \epsilon^{5}+8 b_{1} k R^{2} \alpha \lambda^{3} \epsilon^{3}-32 b_{1} k R^{2} \alpha \lambda^{3} \mu^{2} \epsilon^{3}+$ $92 b_{2} k R^{3} \alpha \lambda^{3} \mu \epsilon^{3}-a_{0} b_{1} R \alpha \lambda \mu^{2} \epsilon^{2}-b_{1} f R \alpha \lambda \mu^{2} \epsilon^{2}-3 a_{2} b_{1} R^{3} \alpha \lambda \epsilon^{2}-3 a_{1} b_{2} R^{3} \alpha \lambda \epsilon^{2}+$ $a_{0} b_{1} R \alpha \lambda \epsilon^{2}+b_{1} f R \alpha \lambda \epsilon^{2}+4 a_{1} b_{1} R^{2} \alpha \lambda \mu \epsilon^{2}+4 a_{0} b_{2} R^{2} \alpha \lambda \mu \epsilon^{2}+4 b_{2} f R^{2} \alpha \lambda \mu \epsilon^{2}-b_{1} R \mu^{2} h \epsilon^{2}+$ $b_{1} R h \epsilon^{2}+4 b_{2} R^{2} \mu h \epsilon^{2}+3 b_{1} k R^{2} \alpha \lambda^{3} \mu^{2} \epsilon-11 b_{2} k R^{3} \alpha \lambda^{3} \mu \epsilon+a_{2} b_{1} R^{3} \alpha \lambda+a_{1} b_{2} R^{3} \alpha \lambda-$ $a_{1} b_{1} R^{2} \alpha \lambda \mu-a_{0} b_{2} R^{2} \alpha \lambda \mu-b_{2} f R^{2} \alpha \lambda \mu-b_{2} R^{2} \mu h=0$.

(x) $-48 b_{2} k R^{2} \alpha \lambda^{3} \epsilon^{5}-24 b_{1} k R \alpha \lambda^{3} \mu^{3} \epsilon^{5}+144 b_{2} k R^{2} \alpha \lambda^{3} \mu^{2} \epsilon^{5}+24 b_{1} k R \alpha \lambda^{3} \mu \epsilon^{5}+28 b_{2} k R^{2} \alpha \lambda^{3} \epsilon^{3}+$ $12 b_{1} k R \alpha \lambda^{3} \mu^{3} \epsilon^{3}-100 b_{2} k R^{2} \alpha \lambda^{3} \mu^{2} \epsilon^{3}-12 b_{1} k R \alpha \lambda^{3} \mu \epsilon^{3}-2 a_{1} b_{1} R \alpha \lambda \mu^{2} \epsilon^{2}-2 a_{0} b_{2} R \alpha \lambda \mu^{2} \epsilon^{2}-$ $2 b_{2} f R \alpha \lambda \mu^{2} \epsilon^{2}-4 a_{2} b_{2} R^{3} \alpha \lambda \epsilon^{2}+2 a_{1} b_{1} R \alpha \lambda \epsilon^{2}+2 a_{0} b_{2} R \alpha \lambda \epsilon^{2}+2 b_{2} f R \alpha \lambda \epsilon^{2}+6 a_{2} b_{1} R^{2} \alpha \lambda \mu \epsilon^{2}+$ $6 a_{1} b_{2} R^{2} \alpha \lambda \mu \epsilon^{2}-2 b_{2} R \mu^{2} h \epsilon^{2}+2 b_{2} R h \epsilon^{2}+6 b_{2} k R^{2} \alpha \lambda^{3} \mu^{2} \epsilon+a_{2} b_{2} R^{3} \alpha \lambda-a_{2} b_{1} R^{2} \alpha \lambda \mu-$ $a_{1} b_{2} R^{2} \alpha \lambda \mu=0$.

In the equations above, $f=f(t)=\int F(t) d t, \alpha=\alpha(t)$, and $h=h(t)$. Solving the previous system with the aid of a computer, we obtain many solutions to (1.3). These solutions may be obtained from (1.5) and are given by (3.9)-(3.19). In these formulas, $H(t)=\int h(t) d t$ and $\lambda, a_{0}$ are arbitrary parameters. In a formula containing $\sqrt{R}$, we suppose that $R>0$ and if the expression $\sqrt{-R}$ appears, we choose $R<0$. If a formula involves $\sqrt{-R}$ and $\sqrt{1-\mu^{2}}$ (see, e.g., (3.17)) we consider that $R<0$ and $|\mu| \leq 1$.

First Group. $\epsilon=1$ :

(i) $a_{2}=b_{1}=b_{2}=0, a_{1}=6 k \lambda^{2}, h(t)=-\lambda \alpha(t)\left(a_{0}+\int F(t) d t-R k \lambda^{2}\right), \mu=-1$ :

$$
\begin{aligned}
& u_{1}(x, t)=\int F(t) d t+a_{0}-\frac{6 k \lambda^{2} R}{1-\sin (\sqrt{R}(\lambda x+H(t)))}, \\
& u_{2}(x, t)=\int F(t) d t+a_{0}-\frac{6 k \lambda^{2} R}{1-\cos (\sqrt{R}(\lambda x+H(t)))} \\
& u_{3}(x, t)=\int F(t) d t+a_{0}-\frac{6 k \lambda^{2} R}{1-\cosh (\sqrt{-R}(\lambda x+H(t)))} .
\end{aligned}
$$

(ii) $a_{2}=b_{1}=b_{2}=0, a_{1}=-6 k \lambda^{2}, h(t)=-\lambda \alpha(t)\left(a_{0}+\int F(t) d t-R k \lambda^{2}\right), \mu=1$ :

$$
\begin{aligned}
& u_{4}(x, t)=\int F(t) d t+a_{0}-\frac{6 k \lambda^{2} R}{1+\sin (\sqrt{R}(\lambda x+H(t)))} \\
& u_{5}(x, t)=\int F(t) d t+a_{0}-\frac{6 k \lambda^{2} R}{1+\cos (\sqrt{R}(\lambda x+H(t)))}
\end{aligned}
$$




$$
u_{6}(x, t)=\int F(t) d t+a_{0}-\frac{6 k \lambda^{2} R}{1+\cosh (\sqrt{-R}(\lambda x+H(t)))}
$$

(iii) $a_{1}=-6 k \lambda^{2} \mu, b_{1}=0, a_{2}=6 k \lambda^{2}\left(\mu^{2}-1\right) / R, b_{2}=6 k \lambda^{2} \sqrt{R\left(1-\mu^{2}\right)} / R, h(t)=-\lambda \alpha(t)\left(a_{0}+\right.$ $\left.\int F(t) d t-R k \lambda^{2}\right), \mu=\mu$ :

$$
\begin{aligned}
u_{7}(x, t)= & \int F(t) d t+a_{0} \\
& -\frac{6 k \lambda^{2} R\left(1+\mu \cos (\sqrt{R}(\lambda x+H(t)))-\sqrt{1-\mu^{2}} \sin (\sqrt{R}(\lambda x+H(t)))\right)}{\mu^{2}+2 \mu \cos (\sqrt{R}(\lambda x+H(t)))+\cos ^{2}(\sqrt{R}(\lambda x+H(t)))}, \\
u_{8}(x, t)= & \int F(t) d t+a_{0} \\
& -\frac{6 k \lambda^{2} R\left(1+\mu \cosh (\sqrt{-R}(\lambda x+H(t)))-\sqrt{\mu^{2}-1} \sinh (\sqrt{-R}(\lambda x+H(t)))\right)}{(\mu+\cosh (\sqrt{-R}(\lambda x+H(t))))^{2}}, \\
u_{9}(x, t)= & \int F(t) d t+a_{0} \\
& -\frac{6 \lambda^{2} k R\left(1+\mu \sin (\sqrt{R}(\lambda x+H(t)))-\sqrt{1-\mu^{2}} \cos (\sqrt{R}(\lambda x+H(t)))\right)}{1+\mu^{2}+2 \mu \sin (\sqrt{R}(\lambda x+H(t)))-\cos ^{2}(\sqrt{R}(\lambda x+H(t)))} .
\end{aligned}
$$

(iv) $a_{1}=-6 k \lambda^{2} \mu, b_{1}=0, a_{2}=6 k \lambda^{2}\left(\mu^{2}-1\right) / R, b_{2}=-6 k \lambda^{2} \sqrt{R\left(1-\mu^{2}\right)} / R, h(t)=$ $-\lambda \alpha(t)\left(a_{0}+\int F(t) d t-R k \lambda^{2}\right), \mu=\mu$ :

$$
\begin{aligned}
u_{10}(x, t)= & \int F(t) d t+a_{0} \\
& -\frac{6 k \lambda^{2} R\left(1+\mu \cos (\sqrt{R}(\lambda x+H(t)))+\sqrt{1-\mu^{2}} \sin (\sqrt{R}(\lambda x+H(t)))\right)}{\mu^{2}+2 \mu \cos (\sqrt{R}(\lambda x+H(t)))+\cos ^{2}(\sqrt{R}(\lambda x+H(t)))}, \\
u_{11}(x, t)= & \int F(t) d t+a_{0} \\
& -\frac{6 k \lambda^{2} R\left(1+\mu \cosh (\sqrt{-R}(\lambda x+H(t)))+\sqrt{\mu^{2}-1} \sinh (\sqrt{-R}(\lambda x+H(t)))\right)}{(\mu+\cosh (\sqrt{-R}(\lambda x+H(t))))^{2}} .
\end{aligned}
$$


Figure 1 shows solution $u_{11}(x, t)$ for the choices $\mu=1.3, R=-2.3, k=2.3, \lambda=1.9$, $a_{0}=1, F(t)=\sin (t), \alpha(t)=t,-3 \leq x \leq 4$, and $-1 \leq t \leq 1.1$ :

$$
\begin{aligned}
u_{12}(x, t)= & \int F(t) d t+a_{0} \\
& -\frac{6 k \lambda^{2} R\left(1+\mu \sin (\sqrt{R}(\lambda x+H(t)))+\sqrt{1-\mu^{2}} \cos (\sqrt{R}(\lambda x+H(t)))\right)}{1+\mu^{2}+2 \mu \sin (\sqrt{R}(\lambda x+H(t)))-\cos ^{2}(\sqrt{R}(\lambda x+H(t)))} .
\end{aligned}
$$

Figure 2 shows solution $u_{12}(x, t)$ for the choices $\mu=0.3, R=2.3, k=2.3, \lambda=1.9, a_{0}=1$, $F(t)=\sin (t), \alpha(t)=t,-3 \leq x \leq 4$, and $-1 \leq t \leq 1.1$.

Second Group. $\epsilon=-1$ :

(v) $a_{1}=b_{1}=0, a_{2}=6 k \lambda^{2} / R, b_{2}=-6 k \lambda^{2} / \sqrt{-R}, h(t)=-\lambda \alpha(t)\left(a_{0}+\int F(t) d t+R k \lambda^{2}\right)$, $\mu=0$ :

$$
u_{13}(x, t)=\int F(t) d t+a_{0}+\frac{6 k \lambda^{2} R(1+\sin (\sqrt{-R}(\lambda x+H(t))))}{\cos ^{2}(\sqrt{-R}(\lambda x+H(t)))} .
$$

(vi) $a_{1}=b_{1}=0, a_{2}=6 k \lambda^{2} / R, b_{2}=6 k \lambda^{2} / \sqrt{-R}, h(t)=-\lambda \alpha(t)\left(a_{0}+\int F(t) d t+R k \lambda^{2}\right), \mu=0$ :

$$
u_{14}(x, t)=\int F(t) d t+a_{0}+\frac{6 k \lambda^{2} R(1-\sin (\sqrt{-R}(\lambda x+H(t))))}{\cos ^{2}(\sqrt{-R}(\lambda x+H(t)))} .
$$

(vii) $a_{1}=6 k \lambda^{2} \mu, a_{2}=6 k \lambda^{2}\left(1-\mu^{2}\right) / R, b_{1}=0, b_{2}=6 k \lambda^{2} \sqrt{R\left(\mu^{2}-1\right)} / R, h(t)=-\lambda \alpha(t)\left(a_{0}+\right.$ $\left.\int F(t) d t+R k \lambda^{2}\right), \mu=\mu$ :

$$
\begin{aligned}
u_{15}(x, t)= & \int F(t) d t+a_{0}+\frac{6 k \lambda^{2} \mu R}{\mu+\cosh (\sqrt{R}(\lambda x+H(t)))} \\
& +\frac{6 k \lambda^{2} R\left(1-\mu^{2}+\sqrt{\mu^{2}-1} \sinh (\sqrt{R}(\lambda x+H(t)))\right)}{(\mu+\cosh (\sqrt{R}(\lambda x+H(t))))^{2}}, \\
u_{16}(x, t)= & \int F(t) d t+a_{0}+\frac{6 k \lambda^{2} \mu R}{\mu+\cos (\sqrt{-R}(\lambda x+H(t)))} \\
& +\frac{6 k \lambda^{2} R\left(1-\mu^{2}+\sqrt{1-\mu^{2}} \sin (\sqrt{-R}(\lambda x+H(t)))\right)}{\mu^{2}+2 \mu \cos (\sqrt{-R}(\lambda x+H(t)))+\cos ^{2}(\sqrt{-R}(\lambda x+H(t)))} .
\end{aligned}
$$




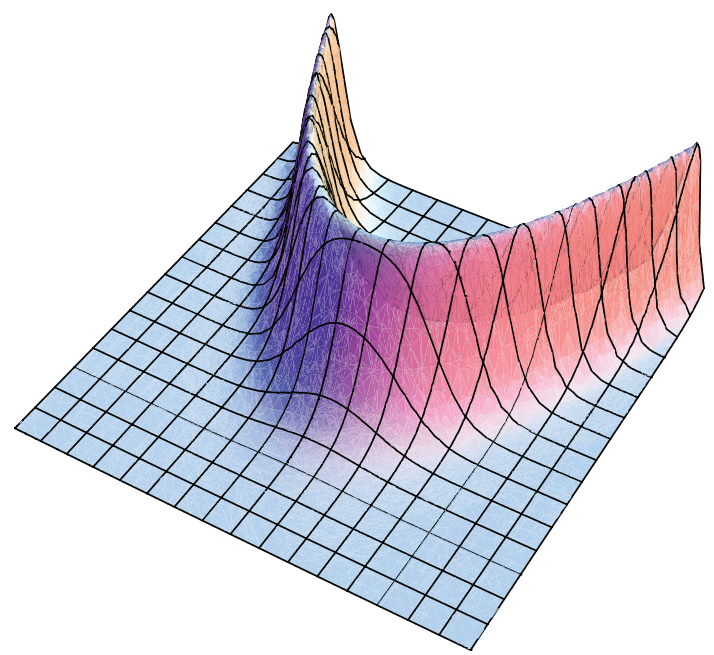

Figure 1: Graphic of solution $u_{11}(x, t),-3 \leq x \leq 4$ and $-1 \leq t \leq 1.1$.

(viii) $a_{1}=6 k \lambda^{2} \mu, a_{2}=-6 k \lambda^{2}\left(1-\mu^{2}\right) / R, b_{1}=0, b_{2}=-6 k \lambda^{2} \sqrt{R\left(\mu^{2}-1\right)} / R, h(t)=$ $-\lambda \alpha(t)\left(a_{0}+\int F(t) d t+R k \lambda^{2}\right), \mu=\mu$ :

$$
\begin{aligned}
u_{17}(x, t)= & \int F(t) d t+a_{0}+\frac{6 k \lambda^{2} \mu R}{\mu+\cosh (\sqrt{R}(\lambda x+H(t)))} \\
& +\frac{6 k \lambda^{2} R\left(1-\mu^{2}-\sqrt{\mu^{2}-1} \sinh (\sqrt{R}(\lambda x+H(t)))\right)}{(\mu+\cosh (\sqrt{R}(\lambda x+H(t))))^{2}}, \\
u_{18}(x, t)= & \int F(t) d t+a_{0}+\frac{6 k \lambda^{2} \mu R}{\mu+\cos (\sqrt{-R}(\lambda x+H(t)))} \\
& +\frac{6 k \lambda^{2} R\left(1-\mu^{2}-\sqrt{1-\mu^{2}} \sin (\sqrt{-R}(\lambda x+H(t)))\right)}{\mu^{2}+2 \mu \cos (\sqrt{-R}(\lambda x+H(t)))+\cos ^{2}(\sqrt{-R}(\lambda x+H(t)))} .
\end{aligned}
$$

\subsection{Rational Solutions}

We seek rational solutions to (1.8) $(R=\mu=0)$ in the form given by (3.6) with $m=2$,

$$
V(\xi)=\sum_{i=0}^{2} a_{i} \tau^{i}(\xi)
$$




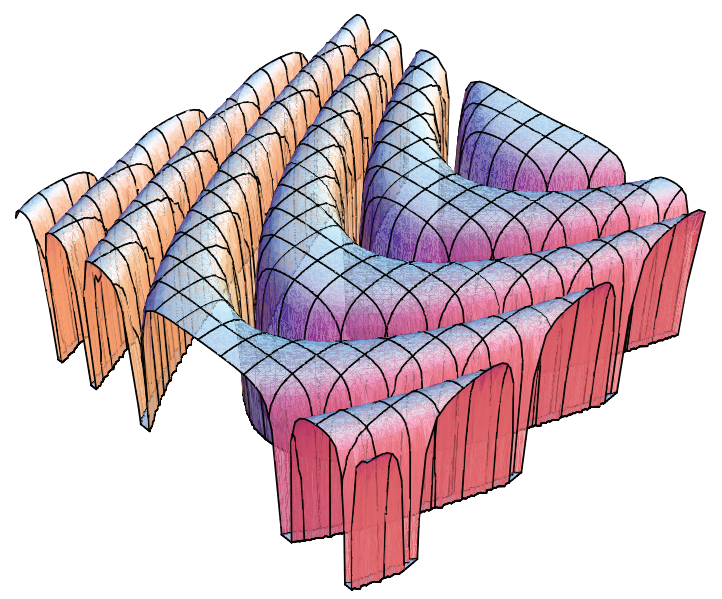

Figure 2: Graphic of solution $u_{12}(x, t),-3 \leq x \leq 4$ and $-1 \leq t \leq 1.1$.

where

$$
\tau^{\prime}(\xi)=\tau^{2}(\xi)
$$

Note that this last equation has the general solution

$$
\tau(\xi)=-\frac{1}{\xi+C} .
$$

We now substitute (3.20) into (1.8), and using the relation $\tau^{\prime}(\xi)=\tau^{2}(\xi)$ we obtain an equation whose left-hand side is a polynomial in the variable $\tau=\tau(\xi)$. We equate each coefficient of this polynomial to zero and we get the following algebraic system.

(i) $h(t) a_{1}+\alpha(t) \lambda\left(\int F(t) d t\right) a_{1}+\alpha(t) \lambda a_{0} a_{1}=0$.

(ii) $\alpha(t) \lambda a_{1}^{2}+2 h(t) a_{2}+2 \alpha(t) \lambda\left(\int F(t) d t\right) a_{2}+2 \alpha(t) \lambda a_{0} a_{2}=0$.

(iii) $6 k a_{1} \lambda^{3}+3 a_{1} a_{2} \lambda=0$.

(iv) $24 k a_{2} \lambda^{3}+2 a_{2}^{2} \lambda=0$.

Solving this system gives $a_{0}=a_{0}, a_{1}=0, a_{2}=-12 k \lambda^{2}$ and

$$
h(t)=-\lambda \alpha(t)\left(\int F(t) d t+a_{0}\right) .
$$

A rational solution of (1.8) is given by

$$
V(\xi)=a_{0}-\frac{12 k \lambda^{2}}{(\xi+C)^{2}}
$$


According to (1.5), (1.6), and (1.7) we obtain the following rational solution to (1.3):

$$
u_{19}(x, t)=\int F(t) d t+a_{0}-\frac{12 k \lambda^{2}}{\left(\lambda x-\int\left(\lambda \alpha(t)\left(\int F(t) d t+a_{0}\right)\right) d t+C\right)^{2}} .
$$

\section{Conclusions}

In this paper, by using the projective Riccati equation and the Exp-function methods, with the help of a symbolic computation engine, we obtain exact solutions for a generalized KdV equation with forcing term (1.3). The methods certainly works well for a large class of very interesting nonlinear equations. The main advantage of these methods is their capability of greatly reducing the size of computational work compared to existing techniques such as the pseudospectral method, the inverse scattering method, Hirota's bilinear method, and the truncated Painlevé expansion. The Exp-function method gives us more general solutions with some free parameters than the projective Riccati equation method. It also has other interesting applications. For instance, the Exp-function method may be applied not only to differential equations but also to differential-difference equations or Stochastic equations [32-35].

\section{References}

[1] N. Nirmala, M. J. Vedan, and B. V. Baby, "Auto-Bäcklund transformation, Lax pairs, and Painlevé property of a variable coefficient Korteweg-de Vries equation," Journal of Mathematical Physics, vol. 27, no. 11, pp. 2640-2646, 1986.

[2] E. Fan and Y. C. Hon, "Generalized tanh method extended to special types of nonlinear equations," Zeitschrift fur Naturforschung A, vol. 57, no. 8, pp. 692-700, 2002.

[3] C. A. Gómez, "Exact solutions for a new fifth-order integrable system," Revista Colombiana de Matemáticas, vol. 40, no. 2, pp. 119-125, 2006.

[4] C. A. Gomez and A. H. Salas, "Exact solutions for a reaction diffusion equation by using the generalized tanh method," Scientia et Technica, vol. 13, no. 35, pp. 409-410, 2007.

[5] C. A. Gómez, A. H. Salas, and B. Acevedo Frias, "New periodic and soliton solutions for the generalized BBM and Burger's-BBM equations," Applied Mathematics and Computation. In press.

[6] C. A. Gómez and A. H. Salas, "Exact solutions for a new integrable system (KdV6)," Journal of Mathematical Sciences: Advances and Applications, vol. 1, no. 2, pp. 401-413, 2008.

[7] A. H. Salas, C. A. Gómez, and G. Escobar, "Exact solutions for the general fifth order KdV equation by the extended tanh method," Journal of Mathematical Sciences: Advances and Applications, vol. 1, no. 2, pp. 305-310, 2008.

[8] C. A. Gómez, "A new travelling wave solution of the Mikhailov-Novikov-Wang system using the extended tanh method," Boletín de Matemáticas, vol. 14, no. 1, pp. 38-43, 2007.

[9] A.-M. Wazwaz, "The extended tanh method for new solitons solutions for many forms of the fifthorder KdV equations," Applied Mathematics and Computation, vol. 184, no. 2, pp. 1002-1014, 2007.

[10] C. A. Gómez, "Special forms of the fifth-order KdV equation with new periodic and soliton solutions," Applied Mathematics and Computation, vol. 189, no. 2, pp. 1066-1077, 2007.

[11] C. A. Gómez and A. H. Salas, "The generalized tanh-coth method to special types of the fifth-order KdV equation," Applied Mathematics and Computation, vol. 203, no. 2, pp. 873-880, 2008.

[12] A. H. Salas and C. A. Gómez, "Computing exact solutions for some fifth KdV equations with forcing term," Applied Mathematics and Computation, vol. 204, no. 1, pp. 257-260, 2008.

[13] C. A. Gómez and A. H. Salas, "The variational iteration method combined with improved generalized tanh-coth method applied to Sawada-Kotera equation," Applied Mathematics and Computation. In press.

[14] J.-H. He and X.-H. Wu, "Exp-function method for nonlinear wave equations," Chaos, Solitons $\mathcal{E}$ Fractals, vol. 30, no. 3, pp. 700-708, 2006.

[15] S. Zhang, "Exp-function method exactly solving the KdV equation with forcing term," Applied Mathematics and Computation, vol. 197, no. 1, pp. 128-134, 2008. 
[16] J.-H. He and L.-N. Zhang, “Generalized solitary solution and compacton-like solution of the JaulentMiodek equations using the Exp-function method," Physics Letters A, vol. 372, no. 7, pp. 1044-1047, 2008.

[17] S. Zhang, "Application of Exp-function method to a KdV equation with variable coefficients," Physics Letters A, vol. 365, no. 5-6, pp. 448-453, 2007.

[18] A. H. Salas, "Exact solutions for the general fifth KdV equation by the exp function method," Applied Mathematics and Computation, vol. 205, no. 1, pp. 291-297, 2008.

[19] A. H. Salas, C. A. Gómez, and J. Castillo, "New abundant solutions for the Burger's equation," Computers \& Mathematics with Applications, vol. 58, pp. 514-520, 2009.

[20] C. A. Gómez and A. H. Salas, "The Cole-Hopf transformation and improved tanh-coth method applied to new integrable system (KdV6)," Applied Mathematics and Computation, vol. 204, no. 2, pp. 957-962, 2008.

[21] R. Conte and M. Musette, "Link between solitary waves and projective Riccati equations," Journal of Physics. A, vol. 25, no. 21, pp. 5609-5623, 1992.

[22] A. H. Salas, "Some solutions for a type of generalized Sawada-Kotera equation," Applied Mathematics and Computation, vol. 196, no. 2, pp. 812-817, 2008.

[23] Z. Yan, "The Riccati equation with variable coefficients expansion algorithm to find more exact solutions of nonlinear differential equations," MMRC, AMSS, Academis Sinica, vol. 22, pp. 275-284, 2003.

[24] E. Yomba, "The general projective Riccati equations method and exact solutions for a class of nonlinear partial differential equations," Chinese Journal of Physics, vol. 43, no. 6, pp. 991-1003, 2005.

[25] C. A. Gómez and A. H. Salas, "Special forms of Sawada-Kotera equation with periodic and soliton solutions," International Journal of Applied Mathematical Analysis and Applications, vol. 3, no. 1, pp. 45$51,2008$.

[26] Y. Shang, Y. Huang, and W. Yuan, "New exact traveling wave solutions for the Klein-GordonZakharov equations," Computers \& Mathematics with Applications, vol. 56, no. 5, pp. 1441-1450, 2008.

[27] C. A. Gómez and A. H. Salas, "Exact solutions for the generalized shallow water wave equation by the general projective Riccati equations method," Boletín de Matemáticas, vol. 13, no. 1, pp. 50-56, 2006.

[28] C. A. Gómez and A. H. Salas, "New exact solutions for the combined sinh-cosh-Gordon equation," Lecturas Matematicas, vol. 27, pp. 87-93, 2006.

[29] C. A. Gómez, "New exact solutions of the Mikhailov-Novikov-Wang system," International Journal of Computer, Mathematical Sciences and Applications, vol. 1, pp. 137-143, 2007.

[30] Y. Chen and B. Li, "General projective Riccati equation method and exact solutions for generalized KdV-type and KdV-Burgers-type equations with nonlinear terms of any order," Chaos, Solitons $\mathcal{E}$ Fractals, vol. 19, no. 4, pp. 977-984, 2004.

[31] Taogetusang and Sirendaoerji, "The Jacobi elliptic function-like exact solutions to two kinds of KdV equations with variable coefficients and KdV equation with forcible term," Chinese Physics, vol. 15, no. 12 , pp. 2809-2818, 2006.

[32] S.-D. Zhu, "Exp-function method for the Hybrid-Lattice system," International Journal of Nonlinear Sciences and Numerical Simulation, vol. 8, no. 3, pp. 461-464, 2007.

[33] S.-D. Zhu, "Exp-function method for the discrete mKdV lattice," International Journal of Nonlinear Sciences and Numerical Simulation, vol. 8, no. 3, pp. 465-468, 2007.

[34] C. Dai, X. Cen, and S. S. Wu, "The application of He's exp-function method to a nonlinear differentialdifference equation," Chaos, Solitons \& Fractals, vol. 41, no. 1, pp. 511-515, 2009.

[35] C.-Q. Dai and J.-F. Zhang, "Application of he's EXP-function method to the stochastic mKdV equation," International Journal of Nonlinear Sciences and Numerical Simulation, vol. 10, no. 5, pp. 675$680,2009$. 


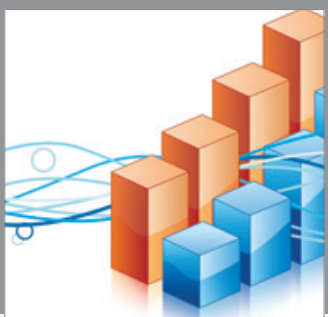

Advances in

Operations Research

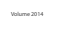

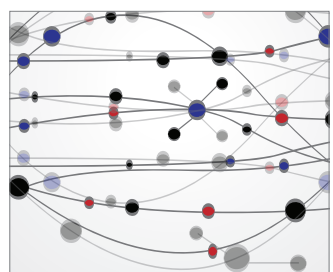

\section{The Scientific} World Journal
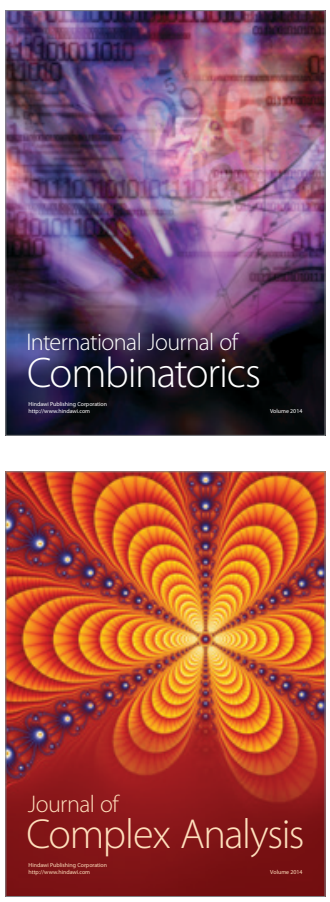

International Journal of

Mathematics and

Mathematical

Sciences
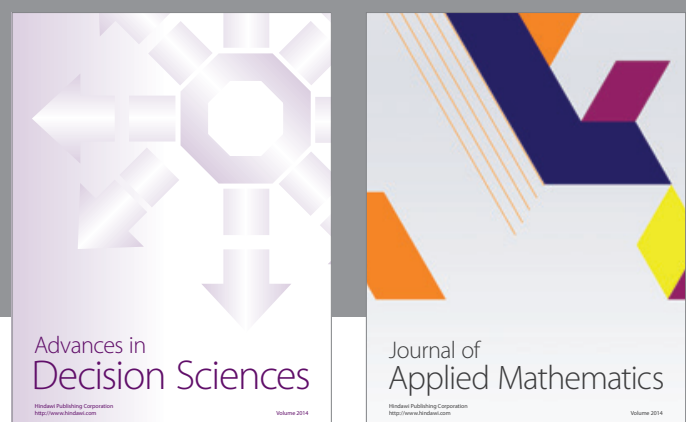

Journal of

Applied Mathematics
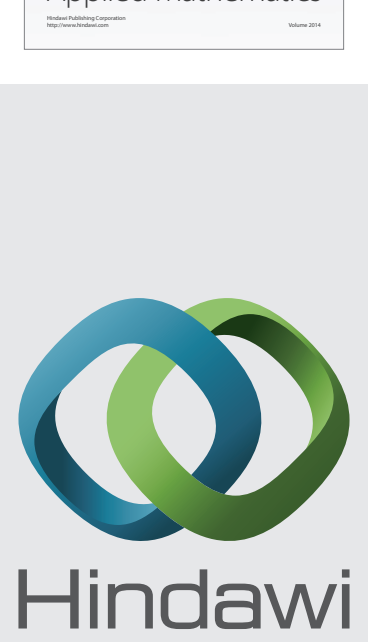

Submit your manuscripts at http://www.hindawi.com
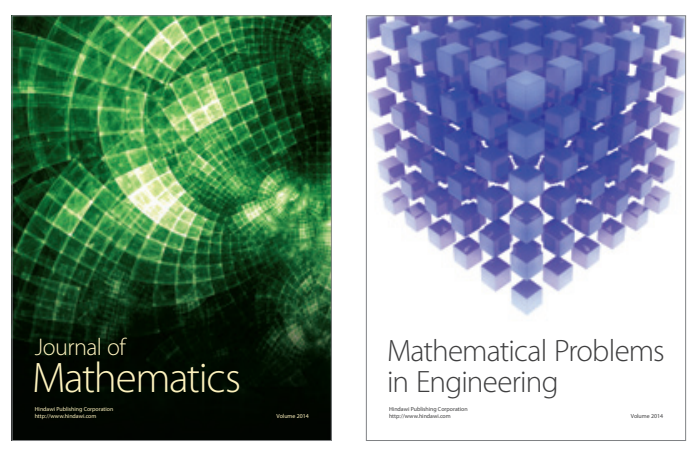

Mathematical Problems in Engineering
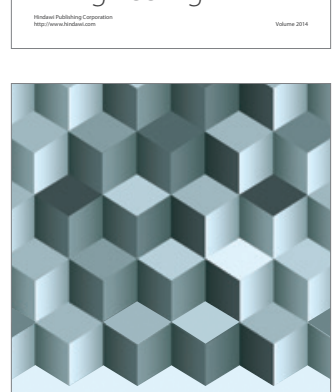

Journal of

Function Spaces
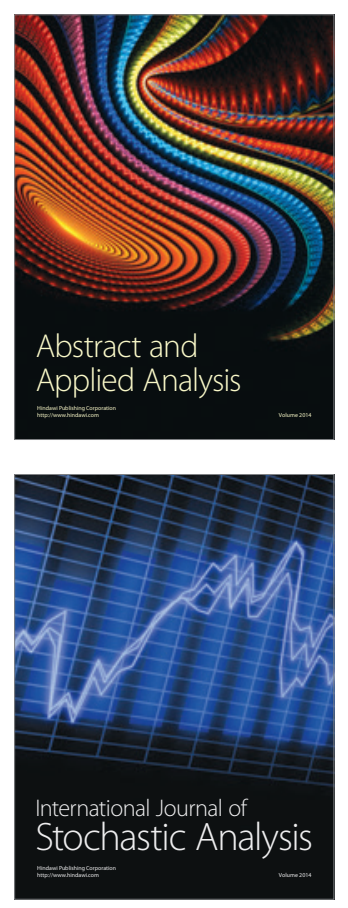

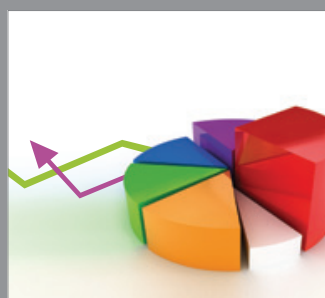

ournal of

Probability and Statistics

Promensencen
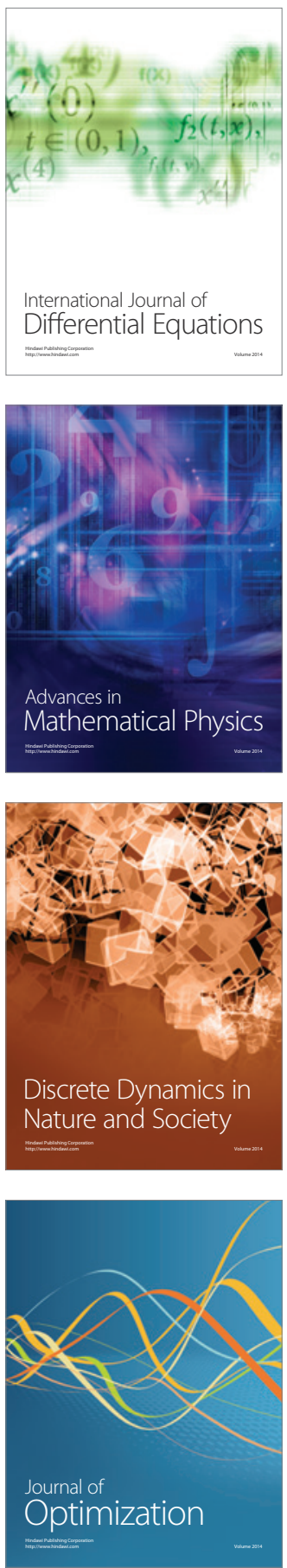